\title{
Of Kings and Cobots
}

In the previous paper and video presentation a consideration of Amazon as a market place of some authority was made. The primary source there and in the course of this series is Brian Dumaine's book Bezonomics (Dumaine 2020). I want to acknowledge that influence especially for matters of fact here. In this series I have put my own construction on an extremely good compilation of detail on Amazon by Dumaine. Other figures are cited when the source is not Dumaine.

The argument so far is that Amazon, the creation of Jeff Bezos, is an entity of a scale and compass to invite comparison with organisations bigger than the company and, in some respects such as the trust of the client in Amazon, like the state. To what and to whom might this kind of trust in a person or an entity or a process such as that described as Bezonomics be compared?

Another question, if we are going to talk about Amazon and state-like entities, is to define what we mean by the word 'state'. Here the word 'state' could refer to a general condition, an order, perhaps public, of matters and to a comparability with the nation state. Regarding the latter, here there is a sense in which the phrases, 'the early state' or 'the incipient state' might apply. We might speak of Amazon as a state about to happen the exact nature of which we do not know but we might start to explore...

Professor Peter Cleave is a Research Associate in the Faculty of Health and Environmental Sciences (AUT University), a Visiting Fellow in Te Ara

Poutama (AUT University), a Member of Common Room, Wolfson College (Oxford University), and a Broadcaster in Te Reo Maori at Kia Ora FM. 
Coming back to trust might we compare the trust people have in Bezos and Amazon with another individual and a tech giant like Bill Gates and Microsoft? Or another figure associated with another supply chain like Jack Ma and Alibaba coming out of another country like China? Just as Bezos is not Gates or Ma but similarities might be seen, comparisons with other companies might or might not be productive. Amazon is not Microsoft. Amazon is not Oracle. And so on down the line of companies, big and small. The description is difficult. Amazon is not, in several respects, a tech company. Amazon is not a city. Amazon is not a tribe.

Amazon does not seem, overtly at least, to offer shelter and security but perhaps there is a covert process through Alexa and other systems that offers these in a new form. It has been suggested that Alexa cuts into and exists in and grows in an area not much occupied so far by the state, close to the human body in a doll like form evoking the film Ex Machina (2014) and the book Living Dolls (2002).

Amazon also seems to be curiously de-centred. Is it actually centralised? Does it exist in a specific space? Neither proposition seems to hold. Like the internet itself Amazon is not centred and the questions about New York as a centre considered below would seem to go with that. This also goes with the idea that Bezos himself is not, at least not necessarily a King or Messiah figure, a point of critical or charismatic focus. The de-centring process finds its form in the face of the consumer, in a variety and number of points of reference as Alexa sits in the home making the home the centre or at least the illusion of such. In point of fact Alexa is in the cloud, in the ether, on the net. And one notion intensifies another; it is not just the authority of the market but the feel of the home, of the hearth that grabs the attention of the client, the customer or, perhaps, the citizen and then the feeling, the knowledge that there are supports offstage. 
Above all there is the authority of data and the best ways to use data. This comes down to having the most data and the best logarithms. With this in mind there are several players, Amazon being at the forefront. One thinks of Alibaba, of JD and a few others with considerable if not outrageous, in certain cases and respects, authority. 'In data we trust', say the boffins at Amazon and so does the clientele, the customers, the citizens in this polity of trust.

These players call the shots across a wide spread of experience. The players might be seen as personalities, as Bezos or Gates, or they might be seen as companies or machines. Are we telling the story of technology or of leaders? What is the historiography here? How do algorithms measure time? How, in the world of AI, is weight given to events across time?

There are examples of attempts, some successful, to control Amazon, to place an authority over it. We might turn to an example from Germany in 2019. Germany's Federal Control Office gave a thirty day notice for Amazon to follow before Amazon could close down a site and this notice holds, apparently, around the world. Here we see Amazon and its algorithms being controlled. It is in Amazon's interests to shut down points that interfere with the optimisation of markets for Amazon and to do this quickly. Here we have an authority outside of Amazon and outside of its host state, the USA dictating terms.

Another example might be with Bernie Sanders and the Stop Bezos Act in 2018. This was meant to stop Bad Employers Zeroing Out Subsidies. The first thing to note is just how big a player in the USA is Bezos to have an Act named after him in this way albeit as an acronym. The second and more important substantive matter might be whether Bezos does or does not look after his 
employees in the USA and I leave that hanging simply because, as Dumaine (ibid 248) points out it all gets a little academic as so many jobs might be taken by robots. The real issue might be automation.

Here there is something of a crossroads perhaps. How long will it be before robotic systems prevail in the fulfilment centres. Now there is talk of people working from home to control robots, of drones doing inspection work of the loading and packing processes. If people become redundant in the workplace will they become a kind of consumer farm supported by Universal Basic Income to enjoy the fruits of the factory? Below the word cobotic is explored. Consideration of the The Stop Bezos Act brings that discussion into view.

Yet another example of authority at the nation-state level or between nation-states is that in October 2020 when a Singaporean arbitrator ruled that in India the partner of Amazon had breached arrangements with its local partner, Reliance, by getting involved with a local third party in such a way as to breach Amazon's deal with Reliance. While this seems to be a victory for Amazon this issue points to the conflict between what Ulrich Beck (Beck, 2000) calls cosmopolitan interests and those of the nation-state. The reason cited by Reliance was to do with the Covid pandemic. Curiously this might be one of the few cases where Amazon has been, at least to begin with, disadvantaged by the pandemic, the indoor living aspect having heightened home delivery and the use of Alexa and other Amazon features.

In the examples above we might draw a distinction between those centred in the United States on the one hand such as the Stop Bezos Act and the general position of Lina Khan (Khan, 2017). In both of these there is the question of the authority of the home state, so to speak, the United States of America. On the other hand there are the international examples from India- and, perhaps, 
Singapore- and Germany. We could say that Amazon is in conflict with states in different ways as its algorithms and use of data operate to maximise the situation for Amazon no matter where the place or country involved is.

On the other hand we might look at some developments and suggest that Amazon is taking advantage of the resources of this or that nation- state. It is noteworthy, perhaps, that as Amazon advances in robotics it has based one of its research centres in Germany to take advantage perhaps of the skill sets on offer there.

This raises the question; where should Amazon be based? It does not behove Amazon to be beholden to any given state if the flywheel is to find its best form, a condition without impediments. There is the discussion of New York or elsewhere in various parts of this mini series as a centre for Amazon. Could or should Amazon be based outside of this earthly frame in cyberspace or in outer space? We might note the plans and beginnings of low orbiting satellites which might connect internet to all robotic systems through the Kuiper satellite system. These questions are considered as this mini series goes along.

We could suggest that Amazon, with the emphasis on the customer whoever that might be and wherever the customer might be found, fits the ecology of the nationstate or context that it finds itself in. The EU is a different context to the USA and there is algorithmic adjustment accordingly. This is a little like Sahlins (1958) and the high and low island ecologies of the Pacific and the different cultural features in each including kinship. India is not the USA even if, as seen through Singaporean or other eyes, it ought to be the same. It is always a question of defining the best entry and exit points and strategies in any and all market contexts with the objective of maximising Amazon's interests. Sometimes, as with Ulrick Beck's cosmopolitanism, the terrain might 
be flat and sometimes in specific nation states there might be specific contours to meet, mix and match.

To come at the question of authority in another way, Bezos seems an unlikely King. This is partly to do with his personality which sometimes seems more like the guy next door doing technical stuff in his garage than a monarch. But also because sovereigns are usually defined by their realms. In the case of Amazon the realm is not a country. In fact, the way the algorithms work is to treat any kind of border, be it one of price or distance or anything else, as a challenge to be overcome. The algorithmic state works back to what the algorithm can learn and apply rather than to an accountable political, social or spatial authority. Governance over is governance from a point of origin not from a point of extent, taking us where it may in the most effective fashion, effective in terms of the rationality set into and then learned upon by the algorithm at base.

Later I will come back to the proposition that the context of home and market informs or presages a new form of citizenship. Amazon pervades the lives of members and invites talk of a culture, of a world of satisfaction. The evolution or progress of states and their empires has often involved new combinations- administrative systems and law with the Romans, naval capacity and effective communication with the British in India or the Dutch in Indonesia for example. In this case the new combination is of multiple presences- in the market, in the home, in the cloud, the presence of service getting better through algorithms all the time.

Amazon is a bit like all of the comparisons and contrasts above. It may be seen from various points of view. And each point of view has its own significance, looks at a distinct sovereignty or domain which link or at least sit beside one another in a jumble. It may be that Amazon is at the point where cross fertilisation across diverse areas 
is occurring. For example the systems in Go stores where customers pick up and do not pay at the checkout as their information is logged through their phones may be useful in the Fulfilment Centres. Alexis is said now to be integrated into some twenty thousand devices and, as noted elsewhere in this series, is riding a wave of development in voice recognition whereby voice could replace text in many ways.

Then there is the waist-high robot that uses Alexa and follows people around in the home and, it is imagined, elsewhere fetching and carrying. There is Alexa in the car. There is Alexa in a lapel pin.

Rather than a poorly assorted jumble of features though there now seems to be a set of integers in Amazon, a cross-referencing and cross fertilisation that takes us back to the idea of the multiplier, this time to a kind of quantum effect (cf.Venture City Amazon's City of the Future 2019)

Then there are drones. This is an interesting example as there are openings and closures to consider. At least apparent closures or slowdowns. In the United States drones in the open sky seem to have met opposition. But over in England, in Cambridge, there has been significant development in drone delivery in open space. And now there is the use of drones inside the Fulfilment Centres, especially those areas staffed by robots to provide aerial views. Such views might be directed by staff working from home. So the drone might not work in open sky in one country but might do so in another, might be developed for work in open sky but find a niche inside, a more comfortable niche perhaps given that there is no wind and one imagines few birds or other things that might interfere with low flight over the stacks in the Fulfilment Centres. 
This is also a cross reference or cross fertilisation; drones were developed for delivery but are used for surveillance. The person running a drone or using the information from a drone from their home is also an example of cobotics discussed later. As are the sensors on vests and the wristbands that direct the hands of workers to shelves on a stack. Similarly there are sensors on Xanthus, a developed robotic transporter that can detect vests on humans.

Now it seems to be a matter of attachments to existing vehicles. What add-ons might there be for drive units? One important recent clip-on, inter alia, is the conveyor belt that might be attached to the Pegasus drive unit. And to what else might Alexa be attached (cf Venture City op.cit)?

From one point of view, Prime, Amazon is a rewards programme operating through a company card. But it offers membership which is extremely far reaching and, through Alexa, extremely intimate. Combinations through Prime, the main club, are important Over half of Whole Foods customers are Prime members. Is this the real reason for the purchase of Whole Foods? The combined memberships go with, complement and extend the cross fertilisations suggested above. There is after Clifford Geertz (1973), a thickening of things.

Is this kind of extensive, multi-faceted membership alike to citizenship? You join one club and gain access to another and another, to satisfaction without apparent end. In an interview from 2000 Bezos is backing Tony Hsieh's company Zappos. Hsieh has written a book entitled Delivering Happiness. Bezos is talking about inventing your way out of the box, of inventing on behalf of the customer. Is Amazon a Happiness Club? Amazon acquired Zappos in 2009. 
The emphasis on happiness and satisfaction evoke the work of Neil Postman in his book Amusing ourselves to death (1965) regarding television. The sense of immediate gratification that television gives has echoes in the happiness and satisfaction for the customer that Bezos and Hsieh seek. We might go to Aldous Huxley's Brave New World (1998) while we are at this point.

What kind of tax are people prepared to pay for happiness and satisfaction?

When we speak of Amazon in terms of agency what do we mean? Are we talking about Bezos, about Amazon as a corporate entity or about the algorithms involved? Who or what are the critical actors? Are things in our life caused by Bezos or do we control things with his apparently unlimited help? Or does Amazon simply invite us in to a world of algorithms operating across our fields of experience? In this view the algorithms exist to maximise or happiness, to increase our satisfaction and they form the critical agency.

We might turn to Bezos himself to begin with and ask about agency. One idea is that by virtue of personality and a few key character traits like perseverance, a long term view, small group discourse, tight descriptors like the Six Pager or the Two Pizza rule, an emphasis on the customer in terms of quality and high speed delivery amongst other things Bezos has been the key agent. Other factors like data and algorithms are considered elsewhere in this series but it might be profitable to study what Bezos himself has suggested in various talks.

In a talk at the George Bush Center in 2018 Bezos outlines four key points. The first is customer obsession where the customer is always pulling you along and this is distinct from being competition focused. The second is eagerness to invent. Next there is the long term thinking of 5-7 years and the last is operational standards. Bezos 
also refers to sufficient scale and speaks of a cultural thread. He also talks of having a 'deep keel'. But a critical point may be long term thinking, working in the future.

Regarding the first point, the price is critical and if we turn to space and Bezos' activities there the first order of business is to set up the customer with price. Bezos says in the Bush lecture that he wants to do the "heavy lifting 'to set up the infrastructure so that one trillion people live in space'. This is possibly like getting players, sellers and customers into his market. Once the process described consistently through this mini series starts the algorithms are bought to bear to rationalise sellers and to secure the support of buyers. As he says, he is not trying to sell. His purpose is to 'help someone make a purchase decision'. The price of admission to space is too high right now but once past this challenge the doors will open.

In an interview alongside his brother in 2017 Bezos discusses work-life balance and considers that such a distinction is not useful. Rather he seeks a harmony between work and other things. He speaks of adventure and fellowship with an emphasis on the shared challenge, the shared experience. He also talks about an emphasis on the long term and refers to the 10,000 year clock. Bezos also offers a perspective on Earth and its issues with his vision of long term human expansion into space this being a way forward, a way to save humanity and the planet.

Across these features Bezos has the patience of Job. In almost every case, every workaday example and every grand plan Bezos plans to wait. It may be that partly he riffs off the American weakness for immediate gratification. By playing against that and against the tendencies of the market to aim for immediate release, relief and comprehension, all the while looking at the faults of those around him in the market, he waits them out. At the same time he uses his concepts, his work 
concepts discussed below to stay in the present with his guiding principle that it is always Day One. It is a paradox or a parable of time, to think in the long term and stay in the present.

With space, the latest venture, we find a way to connect to the beginning, the earliest venture with books. Its all about price in the long, sometimes very long term. Bezos, in the interview with his brother argues that the main obstacle in space travel is price because a rocket might only be used once. The problem to be solved is how to use the same parts of or the same rockets to launch into space. With space exploration and, long term, space settlement Bezos has a handle on what to do and now he searches long hard and clearly on how to find reusable utilities. And it's like what he did with books; to send them to the customer quickly and efficiently at the right price. He kept the price lower than others would have for a longer time until he ran a significant corner of the market. If the starting end of space travel is subsidised this may be offset somehow in the longer term. It's not just a matter of driving down the start up costs, it may be necessary to sustain them until a trick might be turned. Again this is what happened with books- speed of delivery and a set of factors helped the bottom line eventually even though his costs were near or over limit for a long time.

To begin the description of key terms employed by Bezos we might start with vocabulary like the word 'flywheel'. We might go back to the work of Jim Morris who influenced Bezos in no small way and just as we might need to clarify the words 'algorithm' and 'flywheel' we might also define the word 'hub'. We might also go to ideas of specific vocabulary, to Pocock's political languages (Pocock 1989), to a set of words that operate as keys to a culture.

So much of the thinking about hubs is in terms of physical space. The city has loomed large in that thinking 
because of the use or road and sea transport to connect with and through cities. This has come to mean that we think of cities both as places for hubs and as hubs themselves. What are hubs in the day of drones and the internet? What are hubs in the day of increased use of airspace and cyberspace?

Who is using what hubs? When? Why? To come back to the idea of the flywheel the hub is simply a point on a continuum with better and faster packing and delivery in mind.

Coming back to the idea of the city there is the sense that the city offered a concentration and an integration of hubs- information hubs, entertainment hubs, distribution and other hubs. Amazon offers a discombobulation of that. The information and entertainment hubs might be in cyberspace on the net, the distribution hubs might be in rural locations serviced, perhaps, by drones that do not go through or over cities. This might take us to an ex-city situation. We might need to go back to the development of the industrialized city and talk of the early state of the Incas or Egyptians to a pre-city situation, if you like, and look to a post-city situation which might so far only be found in fiction like Rob Hart's The Warehouse (2019). So far the Fulfilment Centres have been outside cities and this might seem to cohere with Hart's vision. However, we could look at the Beehive idea that Amazon is developing which involves the use of high rise building, presumably in the cities so that the bottom floors might be shops but the higher floors are ports for drones as well as fulfilment centres ( $c f$. Venture City 2019). Then again, we might envisage simply adding floors to the existing hubs in rural areas. Drones going to the upper floors do not need arterial road or sea links such as might be found in a city situation.

Cities usually require urban dwelling workers. If, for example, a car is assembled, packed and shipped by 
robots with support coming from people working from home or at any distance from the assembly point then the requirements of both the cities and the workers change. If the robots require less and less inputs from humans as time goes by then we may be looking at Universal Basic Income and what I call 'consumer farms' as discussed later. What might such communities look like?

Staying with drones we come to power poles. We might see the power poles being used as recharging stations for drones and also as places that drones could unload packages on a chute that drops to the ground where a Scout, a small driver-less vehicle, might carry on the delivery to the door of the customer where a robot could open the door and carry the item inside. Delivery from factory to gate, porch, kitchen or even the refrigerator is not being done first by road then by hand and a village or cluster of houses with nearby poles or even, given a pandemic, isolated houses with their own poles seems to be the idea here.

Looking at the flywheel from another point of view we might start at the other end, at the satisfaction experienced by the consumer and say that the flywheel works, as do the hubs and the algorithms in terms of the emphasis placed by Bezos on the immediate, on immediate satisfaction with the concept of Day One.

Or we might start with the algorithms themselves which are set to maximise delivery in time and space just as they are set to optimise movement in the warehouse. We could start with tech, thinking about the radio frequency vests. We could think about the limits of technology such as the difficulty robots have with deep bin 'picking' and then proceed to think about the way this might alter processes as with an example not from Amazon but from J D Com in Shanghai China where only standard sized packages are employed in an area of some size which has only four workers. These human workers are cobots. 
Algorithms make cobots of us all. We could go to the film Ex Machina (2014) for a reflection on the word cobot just as we might go to Gaby Wood's book Living Dolls (2002) and ask about control. As Dumaine notes, people even say thank you to a robot (Dumaine ibid180).

The argument so far is that Amazon defies description in many ways. We may find a handle in the terms used such as 'flywheel' and 'Day One'. We may find a handle in AI or algorithms although there is little in our literature and experience for us to grab onto. We may find words that suddenly seem to have new meanings or angles such as 'cobot'.

Amazon is a mercantile entity and invites comparison with other mercantile entities. Amazon is now displacing Google for shopping searches. Is it something like a mercantile city state, like Venice in its day? As an entertainment hub we might think of Amazon in terms of Macao or Monte Carlo. But of course Amazon is not a city and nor is it a city-state. It is a template, a place to stake a claim for a market share. It is, if we take the closest point, Alexa, a voice to inform and an ear to ask.

Earlier empires have run on things like numeracy or literacy or good record keeping or bureaucratic systems. People would say if something doesn't work it doesn't add up. This empire runs on instant or near instant gratification. There are a number of things that Bezos initially did with Amazon that the outfit now does by itself that all seem to lead toward algorithms and they are worth looking at one by one in terms of how they satisfy the customer.

Amazon through its flywheel has used several multipliers and it is important to consider what Bezos and Amazon have walked into, what multipliers they have found rather than directly created. Voice for example. McWhorter, 
cited by Dumaine (ibid 120) is arguing that voice recognition might be a revolutionary tool so that people no longer use keyboards or type. Alexa came along at the moment when this voice recognition revolution, if such it is, started to happen. Bezos and Amazon have found themselves in a space where things happen for them. They are in the zone to be multiplied.

Then there are, as it were, negative multipliersmultipliers of unemployment for example. While there is a scale of workers involved with many more workers at Amazon than in Alphabet a great many may be put out of work or made increasingly cobotic. With the Internet of Things there is self driving cars. Dumaine mentions mass redundancies (ibid 127) and there a direct comparison might be made with the wasteland of The Warehouse (ibid) by Hart. This relates to David Susskind's 'advanced capital' (Susskind 2020).

We can see some of this in the names of companies as with Right Hand Robotics of Mass. And all of this is happening with an eye on universal income. Is a class of unemployed consumers, of passive cobots being created? If so what are the economics of this? Dumaine walks around the idea of Universal Basic Income but does not project into the future. The example of JD in Shanghai China with a large factory and only four workers might be portentous. The collapsing of a need for a human work force seems to have happened there, as mentioned above, with only a few adjustments such as standard sized bins making deep picking possible.

Then there is the question of whose influence is being multiplied in the Amazon context, what multiplications, planned or otherwise are there within the multipliers so to speak? Is a sinofication happening with Amazon? This company started in a given state, the USA. It offered, amongst other things, a platform, a market. Amazon's citizens were US buyers and sellers. Then there were US 
brands produced in China. Now that market is being dominated by sellers with their own brands from outside the USA, from China. If brand and price work they might be taken on byAmazon, bought out or replaced. At least that is the way it has worked to date. Will the prospect of Chinese preponderance in the market change this process?

In terms of sovereignty there might be a parable here, the parable of the Chinese seller, firstly in the American and then in the global Amazon market. But has Amazon become or is it becoming a Chinese market? Before the Chinese manufacturers made articles for sellers on Amazon and after they became the sellers themselves. Bezos encourages this with Dragon Boat and in 2018 one third of sellers on Amazon were Chinese. There have been complaints about Chinese hackers pirating other people's data which is gold in the Amazon universe. Have the Chinese competitors figured out weaknesses in the algorithms?

Putting this another way without reference to cultural or political identity or to sovereignty of a national kind, is understanding the algorithm the key to management now? And is such understanding the way forward with multiplication?

In his Bush seminar Bezos talks of negative and positive reviews in regard to books saying that negative is necessary. Now we might look at the fake reviews on Amazon and the role of Chinese sellers in them and ask how the wheat is to be sorted from the chaff.

Amazon like other extremely large entities operating across the world bends the idea of the nation state. And we might ask what can states learn from Amazon? For example could algorithms be used of in campaigns and elections? Could the US postal service learn from Amazon? Could the Amazon delivery systems subsume 
the state delivery systems? It is said that more people are connected by Amazon than those who have landlines in the USA. Taking a communications position regarding states, the idea being that the state offers a communications hub, questions occur. It would seem that such investigations as the Senate Hearings on Big Tech in November 2020 which were not about learning as much as being about breaking up the big tech entities seem, as before, to go nowhere and to follow the indecision that Khan (Khan 2017), as cited earlier, has described.

There is multiplication and alteration. Here we could consider the hybrid retailer. The way a store is organised will change with less space out front for the physical shop and more out back for the warehousing of online material. The use of cyberspace affects the use of physical space.

When I talk about the algorithmic state what do I mean? I mean a market set and organised by algorithms. I mean a domestic situation organised using algorithms with Alexa as a portal. I mean a cloud that cross references needs and aspirations and uses algorithms to meet a wide range of needs that may eventually include political ones. We might also ask about how algorithms learn from the client with the algorithm becoming key.

Sometimes there is algorithmic fit and at other times there is algorithmic dissonance. The German Example, as set out above is an example of algorithmic dissonance. The algorithmic dog which would normally hunt down the competing product and keep it out of the market is refused from the hunt.

When we consider that algorithms run Alexa we could turn to self and group analysis and think of domestic sovereignty. The algorithmic state extends to mind. Here we might consider again the points about instant 
gratification and reinforcement above. Is this a Skinnerian universe which algorithms expressed through Alexa and other parts of the machine have intensified?

Algorithms talk to each other. There are issues of agency and above I have talked about robots and cobots, about personality and Artificial Intelligence and now it is a matter of working out who runs what, what runs who and what runs what as we figure out agency, governance, control and authority.

How might we best consider the resilience of algorithms? This is a question that depends on the learning capacity of the algorithm and the task to hand as well as constraints. Again something like the German Example shows how the task at hand can be derailed and the power, the immediate power of the algorithm broken or stalled.

And then there is agency and authority. Amazon itself seems to be the authority. People run along Amazon rails, to paraphrase Khan, until Amazon decides where they get off. Or do they? And here is the importance of the German example where Amazon was ordered NOT to shut down one of the operators in 'its' market. But we loop back to a cobotic situation. The dependence on algorithms to find and set the appropriate rails is felt as soon as the market returns or is returned to.

Here there has been an attempt to consider authority and agency. So far it has not yielded a comparability, at least not directly, to Li Min's ritual authority and Kingship in early China (Min 2018). But there are echoes perhaps to the idea of shared memory, of social memory of processes, long and short term that might allow a shape of things to come. That shape may not be a classical civilization as suggested to be the case in China but it may be a shape of authority and agency, a shape, a formation, shadowy as it might be, of a state. 


\section{Bibliography}

Beck, Ulrich British Journal of Sociology No 51 Issue No 3 January/March 2000 pp 79-105

Cleave, Peter The Sovereignty Game Victoria University Press 1989

Collins, J Good to Great, Why Some Companies Make the Leap... and Others Don't Random House Business 2001

Dumaine, Brian Bezonomics How Amazon is changing our lives and what the world is learning from it, Simon and Schuster 2020

Geertz, Clifford, "Thick Description: Toward an Interpretive Theory of Culture", The Interpretation of Cultures: Selected Essays, New York: Basic Books 1973 pp. 3-30

Hart, Rob The Warehouse Bantam Press 2019

Hsieh, Tony, Delivering Happiness, Grand Central Publishing 2011

Khan, Lina M. Amazon's Antitrust Paradox The Yale Law Journal 2017 pp 710-805

McWhorter. John H The Power of Babel: A Natural History of Language New York Times Books/Henry Holt 2001

Min, Li Social Memory and State Formation in Early China, Cambridge University Press 2018

Huxley, Aldous Brave New World, Harper 1998

Pocock, J G A Politics, Language and Time Essays on Political Thought and History University of Chicago Press 1989

Postman Neil Amusing Ourselves to Death: Public Discourse in the Age of Show Business. USA: Penguin 1985

Sahlins, Marshall Social Stratification in Polynesia. Monographs of the American Ethnological Society, 29. Seattle: University of Washington Press 1958

Susskind, David A World Without Work, Technology, Automation and How We Should Respond Penguin 2020 


\section{Articles and Internet References}

Bezos interview 23.07.2000

https:/www.youtube.comwatchv=MvuJCeV3K0o

Ex Machina 2014 Universal Pictures

Venture City Amazon's Robotic Empire: Jeff Bezos' Smart Warehouses Nov 12020 Youtube

George W Bush Provincial Center Forum and Leadership https://www.youtube.com/watch?v=xu6vFIKAUxk Youtube April 272018

Bezos talks with his brother Nov 152017 https: / / www.youtube.com/watch?v=Hq89wYzOjfs

Venture City Amazon's City of the Future Nov 72019 Youtube 